\title{
PAPER
}

\section{Executive dysfunction in subcortical ischaemic vascular disease}

\author{
J H Kramer, B R Reed, D Mungas, M W Weiner, H C Chui
}

J Neurol Neurosurg Psychiatry 2002;72:217-220

See end of article for authors' affiliations

.....................

Correspondence to: Dr J Kramer, University of California, San Francisco Campus, Box 0984-PAC, 401 Parnassus Avenue, San Francisco, CA 94143,

USA; kramer@itsa.ucsf.edu

Received

17 January 2001

In final revised form

17 May 2001

Accepted 25 May 2001

\begin{abstract}
Background: Executive dysfunction has been reported in patients with subcortical-frontal pathology, even in the absence of dementia.

Objective: This study was undertaken to determine if impairments in executive functioning could be found in non-demented patients with subcortical lacunes.

Methods: Cross sectional comparison between older control subjects $(n=27)$ and non-demented patients with one or more subcortical lacunes $(n=12)$. All participants were administered a neuropsychological test battery incorporating three measures of executive functioning, the Stroop interference test, California card sorting test, and the initiation-perseveration subtest of the Mattis dementia rating scale.

Results: No group differences were found on measures of recent verbal memory, language, or spatial ability. Normal controls performed better than patients with lacunes in visual memory. On the Stroop interference test, patients with lacunes performed as well as controls on the colour naming condition but slower on the interference condition. Patients with lacunes also generated fewer correct sorts on the California card sort test and achieved lower scores on the initiation-perseveration subtest. Executive measures were correlated with extent of white matter signal hyperintensity but not number of lacunes. Conclusion: Subcortical ischaemic vascular disease is associated with subtle declines in executive functioning and visual memory, even in non-demented patients. The pattern of cognitive impairment after subcortical lacunes is consistent with models of subcortical-frontal circuits.
\end{abstract}

S ubcortical syndromes can produce subcortical-frontal system dysfunction even in the absence of dementia. Bondi et al, ${ }^{1}$ for example, reported that non-demented patients with Parkinson's disease demonstrated selective deficits on frontal system tasks such as fluency, card sorting, and temporal ordering. Patients with basal ganglia calcifications display a frontal pattern of neuropsychological dysfunction, ${ }^{2}$ and normal adults show basal ganglia and thalamic activation on a conceptual reasoning task. ${ }^{3}$

Non-demented patients with subcortical lacunes may also experience cognitive deficits. Longstreth et $a l^{4}$ found silent lacunes $3 \mathrm{~mm}$ or larger in $20 \%$ of a sample of normal elderly people receiving MR scans as part of a cardiovascular study; those subjects with silent lacunes performed less well on a brief cognitive screening. Non-demented patients with CADASIL have subtle impairments on tasks presumed to involve the frontal lobe such as the Wisconsin card sort test and the trail making test. ${ }^{5}$ These findings are consistent with several studies suggesting that patients with dementia and subcortical lacunes present with disproportionately greater executive dysfunction. $^{6-11}$

The present study was designed to more extensively assess cognitive functioning in older but non-demented patients with subcortical lacunes. According to models of subcorticalfrontal systems, ${ }^{7}$ subcortical lacunar infarcts would disrupt dorsolateral prefrontal-subcortical circuits and produce executive impairment. Cognitive abilities such as spatial perception and language that are primarily mediated by cortical and posterior structures should be relatively spared.

\section{METHODS}

\section{Participants}

Subjects were participants in a programme investigating the relation between cerebrovascular ischaemia and dementia. Study applicants were extensively screened for history or presence of substance misuse; major depression, bipolar affective disorder, and other DSM-IV axis I disorders; neurological disease including head trauma with loss of consciousness greater than 15 minutes, brain tumour, hydrocephalus, cortical stroke, Parkinson's disease, and multiple sclerosis; medical diseases known to affect brain function including $B_{12}$ deficiency, thyroid disease, and liver failure; medications that impair CNS function; and lack of fluency in English. All subjects received a thorough neurological examination, including history and interview with a collateral source. Global functional status for all participants was evaluated with the clinical dementia rating scale (CDR). ${ }^{12} \mathrm{~A}$ CDR of 0 indicates normal functioning.

All participants underwent quantitative brain imaging on a 1.5 Tesla VISION ${ }^{\mathrm{TM}}$ MRI system (Siemens Inc, Iselin, NJ, USA). The MRI protocol consisted of sagittal Tl weighted localiser scans followed by oblique axial double spin echo (DSE) scans $(\mathrm{TR} / \mathrm{TE} 1 / \mathrm{TE} 2=5000 / 20 / 80 \mathrm{~ms}$, inplane resolution $1.0 \times 1.0$ $\mathrm{mm}^{2}, 3 \mathrm{~mm}$ thick, contiguous slices). Axial DSE yielded proton density and T2 weighted MR images that were used for semiautomated image tissue segmentation, as described previously. ${ }^{13}$

Lacunes were operationally defined as small ( $>3 \mathrm{~mm}$ ) areas of the brain with increased signal relative to CSF on proton density MRI in subcortical grey and white matter. Lacunes were differentiated from perivascular spaces (PVS) because only lacunes are hyperintense relative to CSF on proton density images. Isointense lesions on PD MRI at the level of the

Abbreviations: CDR, clinical dementia rating scale; DSE, double spin echo; PVS, perivascular spaces; SIVD, subcortical ischaemic vascular disease; MAS, memory assessment scale list learning; CADASIL, cerebral autosomal dominant arterriopathy with subcortical infarcts and leucoencephalopathy 
Table 1 Mean (SD) demographic variables and neuropsychological test results for the SIVD and control groups

\begin{tabular}{llllll}
\hline Variable & SIVD & Control & t Value & p Value & Eta $^{2}$ \\
\hline Age & $73.73(6.16)$ & $72.82(5.57)$ & 0.46 & NS & 0.001 \\
Education & $15.33(2.61)$ & $15.67(2.62)$ & 0.37 & NS & 0.024 \\
Benton form discrimination & $18.75(2.83)$ & $17.78(3.49)$ & 0.85 & NS & 0.004 \\
Boston naming & $54.00(4.31)$ & $56.30(3.47)$ & 1.77 & NS & 0.096 \\
F-A-S & $32.90(14.77)$ & $41.00(14.00)$ & 1.53 & NS & 0.057 \\
MAS trial 6 & $10.50(1.38)$ & $11.07(1.14)$ & 1.36 & NS & 0.054 \\
MAS 30 minute delay & $10.42(1.38)$ & $10.89(1.09)$ & 1.15 & NS & 0.044 \\
MAS recognition hits & $11.83(0.39)$ & $11.56(0.85)$ & 1.08 & NS & 0.010 \\
MAS recognition false positive & $0.17(0.39)$ & $0.07(0.27)$ & 0.87 & NS & 0.001 \\
Biber trial 5 & $12.17(3.07)$ & $15.00(3.31)$ & 2.52 & $<0.05$ & 0.126 \\
Biber 30 minute delay & $11.17(3.81)$ & $14.63(3.48)$ & 2.79 & $<0.05$ & 0.102 \\
Biber recognition hits & $5.86(0.39)$ & $5.88(0.33)$ & 0.42 & NS & 0.001 \\
Biber recognition false positive & $0.08(0.29)$ & $0.12(0.33)$ & 0.29 & NS & 0.001 \\
Stroop colour naming & $80.91(19.96)$ & $88.81(15.65)$ & 1.34 & NS & 0.042 \\
Stroop interference & $37.42(13.01)$ & $47.00(9.64)$ & 2.52 & $<0.017$ & 0.160 \\
Card sort: number of sorts & $4.58(1.08)$ & $5.31(0.68)$ & 2.65 & $<0.017$ & 0.150 \\
Initiation-perseveration & $34.25(3.25)$ & $36.37(1.76)$ & 2.57 & $<0.017$ & 0.155 \\
\hline & & & & & \\
& & & & &
\end{tabular}

anterior commissure or inferior putamen were termed perivascular spaces; outside that region they were defined as cavitated lacunes if they were $3 \mathrm{~mm}$ at maximum width. Images underwent semiautomated image tissue segmentation for quantification of lacunar volume.

Subjects with $\mathrm{CDR}=0$ were recruited through university dementia clinics and Veterans' Association neurology and radiology services. Subjects from the dementia clinics had previously been recruited as healthy community control subjects. Subjects recruited from the Veterans' Association were identified as potential subjects through chart and scan reviews that identified the presence of a subcortical lacune. None of the patients had sought out evaluations because of concerns about cognitive changes. All subjects with $\mathrm{CDR}=0$ who received the neuropsychological tasks were included in this study. A total of 39 subjects from the University of CaliforniaSan Francisco and University of California-Davis Medical Centers with a CDR of 0 were identified. The subcortical ischaemic vascular disease (SIVD) group comprised 12 patients with one or more subcortical lacunes. This group included subjects who initially enrolled as normal controls and were found to have lacunes on neuroimaging. There were six men and six women with a mean age of 73.7 years. Four patients had one lacune, two had two lacunes, and the remaining six had between three and 10 lacunes. Most patients had lacunes bilaterally. Seven patients had thalamic lacunes, seven had basal ganglia lacunes, four had lacunes in the white matter, and three had lacunes in the internal capsule. The control group comprised 27 participants with a CDR of 0 and who had no lacunes visible on MRI. There were 12 men and 15 women with a mean age of 72.8 years.

Global cognitive functioning was assessed with the Mattis dementia rating scale (DRS). The SIVD group had a mean DRS of 137.3 and the control group had a mean DRS of 139.9. There were no differences between the groups on age, education, or DRS score. Subjects with lacunes had significantly greater volume of white matter signal hyperintensity (lacune group: $3.9 \mathrm{~mm}^{3}$ (SDl.9); control group: $2.5 \mathrm{~mm}^{3} \mathrm{~m}(0.9)$ ). Demographic data are summarised in table 1 .

\section{Procedures}

All subjects were administered a standardised neuropsychological battery assessing language, spatial perception, memory, and executive functioning. Language was assessed with the Boston naming test and the controlled oral word association test. ${ }^{14}$ Spatial perception was assessed with the Benton visual form discrimination test. ${ }^{15}$ The memory assessment scale list learning (MAS) ${ }^{16}$ was used to measure verbal memory. The Biber visual memory test ${ }^{17}$ was used to measure visual memory.

Executive functioning was assessed with the Stroop interference test, the California card sorting test, ${ }^{18}{ }^{19}$ and the initiation-perseveration subtest from the Mattis DRS. The Stroop consists of a colour naming condition where subjects name the colour of coloured patches, and an interference condition where subjects are shown an array of colour names printed in different coloured inks. In the interference condition, subjects must inhibit the overlearned reading response and name the ink colour in which the word was printed. This capacity for inhibition is widely regarded as a key component of executive functioning and has been strongly linked to frontal structures. ${ }^{20}$ The Stroop test used in the present study consisted of cards containing 10 rows of 10 stimuli each. Dependent variables were the number of correct responses in 60 seconds on the colour naming condition, the number of correct responses in 60 seconds on the interference condition, and the number of errors on the interference condition. The California card sorting test was included as an index of concept formation and set shifting. Subjects were presented with six cards and instructed to sort them into two groups of three cards each. Each card varies along several dimensions (for example, size, shape, colour, nature of words printed on cards). Subjects are asked to generate as many different sorts as they can in 4 minutes. There are eight different correct ways of sorting the cards. The dependent measures were the number of correct sorts generated within the time limit and the number of repeated (perseverated) sorts. The initiation-perseveration subtest consists of word generation and motor sequencing tasks.

\section{RESULTS}

Group differences in language, spatial perception, and memory were analyzed using independent samples $t$ tests. The three measures of executive functioning central to this study's hypothesis were also analyzed using $t$ tests but to reduce the risk of a type I error, a Bonferroni correction was applied, setting $\alpha$ at 0.017 .

Mean scores for both groups are summarised in table 1 . There were no significant differences between the SIVD and control groups on Boston naming, controlled oral word association test, spatial perception, and the verbal recall and recognition measures. The SIVD group performed below the level of the control group on recall of the Biber stimuli on trial 5 and again after a delay. The groups performed comparably on the delayed recognition condition. 
On the Stroop test, patients with SIVD made significantly fewer correct responses than the controls on the interference condition, whereas there were no group differences on the simpler colour naming condition. Group differences on the interference condition remained significant even after entering the colour naming condition as a covariate in an analysis of variance (ANOVA). There were no group differences in the number of errors on the interference condition.

Significant group differences were also found on the number of correct sorts generated on the California card sort test. Although both groups performed in the average range, the mean number of correct sorts for the control group was almost one sort more than the SIVD group. Three of the 12 patients with SIVD performed one SD or more below the mean of the control group. There were no group differences in the number of perseverated sorts. Finally, the control group achieved significantly higher scores than the SIVD group on the initiation-perseveration subtest. Half of the 12 patients with SIVD scored I SD or more below the mean of the control group.

We had originally hoped to examine the number and location of lacunes within the SIVD group to see if any relations with behavioural indices could be discerned. Unfortunately, most of our sample had multiple, bilateral lacunes, which would obscure any clear brain-behaviour relations. The number of thalamic lacunes correlated with correct sorts on the California card sort test $(r=-0.71 ; \mathrm{p}=0.01)$ and with the initiation-perseveration score $(r=-0.58, \mathrm{p}<0.05)$. The number of lacunes in the right hemisphere also correlated with the California card sort score $(r=-0.76 ; \mathrm{p}<0.01)$. Number of white matter lacunes was negatively correlated with the Stroop interference condition, but the correlation did not reach statistical significance $(r=-0.48 ; \mathrm{p}=0.11)$. None of the correlations between white matter signal hyperintensity and cognitive ability reached significance for the SIVD group. When data from both groups were analyzed, there were significant correlations between white matter signal hyperintensity and two of the three executive function measures (Stroop interference: $r=-0.46, \mathrm{p}<0.01$; card sort: $r=-0.38$, $\mathrm{p}<0.05$ ) but none of the memory, language, or visuospatial measures. Exploratory analyses were also carried out comparing the six patients with SIVD with one or two lacunes with the six patients with three or more lacunes. None of the comparisons reached significance, although the group with more lacunes tended to perform less well than the group with fewer lacunes.

\section{DISCUSSION}

Results indicate that despite functioning without obvious impairment in the community, non-demented patients with subcortical lacunar infarcts exhibit subtle cognitive deficits relative to control subjects. Executive functioning may be particularly vulnerable. Patients with SIVD were slower than controls on the Stroop interference condition, generated fewer target categories on the California card sort test, and had lower scores on the initiation-perseveration subtest of the DRS. Importantly, these differences were found in a group of patients who performed at control levels on measures of global cognitive ability, verbal memory, confrontation naming, verbal fluency, and visuoperceptual skill. The current findings are consistent with other reports suggesting that subcortical vascular disease can produce cognitive changes in the absence of dementia. ${ }^{212}$

Other studies of subcortical syndromes have suggested that recent memory is often impaired. ${ }^{23}{ }^{24}$ We found differences for visual memory but not for verbal memory. There are at least three possible explanations for the absence of significant differences for verbal memory in our study. Firstly, both of our subject groups were high functioning; all participants had CDR scores of 0 and the mean level of education for both groups was over 15 years. Thus, it is possible that the degree of subcortical pathology, although enough to disrupt executive functioning, was insufficient to produce verbal memory impairment. A second possible explanation is that our sample of patients with SIVD was small, and we lacked sufficient statistical power to detect significant differences. A review of our data indicates that the SIVD group performed almost 0.5 SD below the level of the control group on verbal delayed recall, suggesting that there may be small differences in memory that would have been detected with a larger sample. A third possible explanation for the discrepancy is that the visual memory test is a more novel and difficult task, and thus would be more sensitive to mild, non-specific declines in episodic memory.

The term "executive functioning" has been used to refer to a host of cognitive abilities, including conceptual reasoning, inhibition of overlearned patterns of behaviour, inhibition of responses to salient stimuli, mental flexibility, set shifting, organisational ability, planning, regulation of working memory, and fluency of thought. The Stroop interference condition requires examinees to inhibit an overlearned reading response in order to name the colour of ink in which the word is printed. Patients with SIVD were slower than controls, although there were no group differences in the number of times participants incorrectly read the stimulus item. It is unlikely that the Stroop results can be explained solely on the basis of slower response speed, as there were no group differences on the colour naming condition. However, given that several studies have reported slower information processing in subcortical patients ${ }^{25}{ }^{26}$ and patients with frontal strokes, ${ }^{27}$ direct assessment of processing speed changes and the role of task difficulty in SIVD is warranted.

Subjects with SIVD also demonstrated subtle difficulties with conceptual reasoning, as reflected in their fewer correct sorts on a card sorting task. These data are consistent with the findings of Dimitrov et al who reported that non-demented patients with Parkinson's disease had problems with concept formation. ${ }^{28}$ Verbal fluency, another measure sensitive to subcortical-frontal system pathology, was unimpaired in our sample, although Bondi et al have found that parkinsonian patients have verbal fluency deficits. ${ }^{1}$ As with the memory data, the absence of significant group differences on verbal fluency in our study may be related to our patients being less impaired than those of Bondi et al or to small sample size. Whether additional aspects of executive functioning are compromised in SIVD warrants further study; set shifting deficits, for example, have been reported in other subcortical syndromes, even in the absence of dementia. ${ }^{29}$

Some controversy exists regarding the relation between white matter signal hyperintensities and cognitive function. Wahlund $e t ~ a l^{30}$ found no significant correlations between the degree of or increase in signal hyperintensities and results from any of the neuropsychological tests. By contrast, other studies have suggested a relation in elderly adults without dementia. ${ }^{31-33}$ White matter foci in periventricular and subcortical regions have been found to be related to memory, ${ }^{32-34}$ executive skills, ${ }^{33}{ }^{34}$ and global cognitive ability. ${ }^{33}$ In the present study, the extent of white matter signal hyperintensity was found to be correlated with two of the three executive measures but with none of the other neuropsychological variables.

The presence of a decline in executive functioning without measurable changes in global cognitive abilities, verbal memory, confrontation naming, verbal fluency, or visuoperceptual skill, is most consistent with a subcortical-frontal model for SIVD. However, we cannot state with certainty that frontal structures are definitely affected after subcortical lacunes in the absence of pathological confirmation or in vivo evidence from structural or functional imaging. In other subcortical syndromes, however, metabolic changes in the cerebral cortex have been documented using PET. In patients with multiple sclerosis, for example, there are widespread reductions in cortical metabolism that are most striking in the 
superior mesial frontal cortex and superior dorsolateral frontal cortex. ${ }^{35}$ Similarly, frontal hypoperfusion has been documented in progressive supranuclear palsy and Parkinson's disease. ${ }^{3637}$ Cortical changes in regional cerebral blood flow and cerebral metabolic rate for oxygen have also been reported in patients with dementia or mild cognitive impairment secondary to lacunar infarcts. ${ }^{38-40}$ The patterns of hypoactivity are often generalised without frontal prominence, however, and patients used in these studies are more compromised than those in the present study. Consequently, the evidence that the subtle cognitive changes we found in our patients with lacunes is due to disruption of subcortical-frontal circuits is primarily behavioural and awaits further confirmation.

\section{ACKNOWLEDGEMENTS}

This work was supported by a grant from the National Institute on Aging, POl AG12435 (HCC).

\section{Authors' affiliations}

J H Kramer, San Francisco Medical Center, University of California, 401 Parnassus Avenue, San Francisco, CA 94143, USA

B R Reed, D Mungas, Davis Medical Center, University of California, 150 Muir Rd, Martinez, CA94553, USA

M W Weiner, Veterans' Administration Medical Center, 4150 Clement

St, San Francisco, CA 94121, USA

H C Chui, University of Southern California, 7601 East Imperial

Highway, Downey, CA 90242, USA

\section{REFERENCES}

1 Bondi MW, Kazhiak AW, Bayles KA, et al. Contributions of frontal system dysfunction to memory and perceptual abilities in Parkinson's disease. Neuropsychology 1993;7:89-102.

2 Lopez-Villegas D, Kulisevsky J, Deus J, et al. Neuropsychological alterations in patients with computed tomography-detected basal ganglia calcification. Arch Neurol 1996:53:251-6.

3 Rao SM, Bobholz JA, Hammeke TA, et al. Functional MRI evidence for subcortical participation in conceptual reasoning skills. Neuroreport 1997;8: 1987-93.

4 Longstreth WT Jr, Bernick C, Manolio TA, et al. Lacunar infarcts defined by magnetic resonance imaging of 3660 elderly people: the cardiovascular health study. Arch Neurol 1998;55:1217-25.

5 Taillia H, Chabriat H, Kurtz A, et al. Cognitive alterations in non-demented CADASIL patients. Cerebrovasc Dis 1998;8:97-101.

6 Cummings JL. Vascular subcortical dementias. Dementia 1994:5:177-80.

7 Cummings JL. Anatomic and behavioral aspects of frontal-subcortical circuits. Ann N Y Acad Sci 1995;769.

8 Lamar M, Podell K, Carew TG, et al. Perseverative behavior in Alzheimer's disease and subcortical ischemic vascular dementia. Neuropsychology 1997; 1 1:523-34.

9 Lafosse JM, Reed BR, Mungas D, et al. Fluency and memory differences between ischemic vascular dementia and Alzheimer's disease. Neuropsychology 1997;11:514-22.

10 McPherson SE, Cummings JL. Neuropsychological aspects of vascular dementia. Brain Cogn 1996:31:269-82.

11 Wolfe N, Linn R, Babikian VL, et al. Frontal systems impairment following multiple lacunar infarcts. Arch Neurol 1990;47:129-32.

12 Hughes CP, Berg L, Danziger WL, et al. A new clinical scale for the staging of dementia. Br J Psychiatry 1982;140:566-72.

13 Fein G, Di Sclafani V, Tanabe J, et al. Hippocampal and cortical atrophy predict dementia in subcortical ischemic vascular disease. Neurology 2000;55:1626-35.

14 Benton AL, Hamsher K. Multilingual aphasia examination. lowa City: AJA Associates, 1989.
15 Benton AL, Hamsher K, Varney NR, et al Contributions to neuropsychological assessment. New York: Oxford University Press, 1983.

16 Williams JM. Cognitive behavior rating scale. Odessa: Psychological Assessment Resources, 1991.

17 Glosser G, Goodglass H, Biber C. Assessing visual memory disorders. Psychol Assess 1989;1:82-91.

18 Delis DC, Squire LR, Bihrle A, et al. Componential analysis of problem-solving ability: performance of patients with frontal lobe damage and amnesic patients on a new sorting test. Neuropsychologia 1992; 30:683-97.

19 Delis DC, Kaplan E, Kramer JH. The Delis-Kaplan executive function scale. San Antonio: The Psychological Corporation, 2000.

20 Vendrell P, Junque C, Pujol J, et al. The role of prefrontal regions in the Stroop task. Neuropsychologia 1995;33:341-52

21 O'Connell JE, Gray CS, French JM, et al. Atrial fibrillation and cognitive function: case-control study. J Neurol Neurosurg Psychiatry 1998;65:386-9.

22 Zito M, Muscari A, Marini E, et al. Silent lacunar infarcts in elderly patients with chronic non-valvular atrial fibrillation. Aging (Milano) 1996;8:341-6.

23 Dubois B, Pillon B. Cognitive deficits in Parkinson's disease. J Neurol 1997;244:2-8.

24 Jenkins M, Malloy P, Salloway S, et al. Memory processes in depressed geriatric patients with and without subcortical hyperintensities on MRI. J Neuroimaging 1998;8:20-6.

25 Huber SJ, Paulson GW. Memory impairment associated with progression of Huntington's disease. Cortex 1987;23:275-83.

26 Huber SJ, Shuttleworth EC, Freidenberg DL. Neuropsychological differences between the dementias of Alzheimer's and Parkinson's diseases. Arch Neurol 1989;46:1287-91.

27 Leskela $M$, Hietanen $M$, Kalska $H$, et al. Executive functions and speed of mental processing in elderly patients with frontal or non-frontal ischemic stroke. Eur J Neurol 1999;6:653-61.

28 Dimitrov M, Grafman J, Soares AH, et al. Concept formation and concept shifting in frontal lesion and Parkinson's disease patients assessed with the California card sorting test. Neuropsychology 1999;13:135-43

29 Tamaru F. Disturbances in higher function in Parkinson's disease. Eur Neurol 1997;38:33-6.

30 Wahlund LO, Almkvist $\mathrm{O}$, Basun $\mathrm{H}$, et al. MRI in successful aging, a 5 year follow up study from the 8th to 9th decade of life. Magn Reson Imaging 1996;14:601-8.

31 Baum KA, Schulte C, Girke W, et al. Incidental white-matter foci on MR in "healthy" subjects: evidence of subtle cognitive dysfunction. Neuroradiology 1996:38:755-60

32 de Groot JC, de Leeuw FE, Oudkerk M, et al. Cerebral white matter lesions and depressive symptoms in elderly adults. Arch Gen Psychiatry 2000;57: 1071-6

33 Gunning-Dixon FM, Raz N. The cognitive correlates of white matter abnormalities in normal aging: a quantitative review. Neuropsychology 2000; 14:224-32.

34 Kramer-Ginsberg E, Greenwald BS, Krishnan KR, et al. Neuropsychological functioning and MRI signal hyperintensities in geriatric depression. Am J Psychiatry 1999;156:438-44

35 Bakshi R, Miletich RS, Kinkel PR, et al. High-resolution fluorodeoxyglucose positron emission tomography shows both global and regional cerebral hypometabolism in multiple sclerosis. J Neuroimaging 1998:8:228-34.

36 Karbe H, Grond $M$, Huber $M$, et al. Subcortical damage and cortical dysfunction in progressive supranuclear palsy demonstrated by positron emission tomography. J Neurol 1992;239:98-102.

37 Defebvre L, Lecouffe P, Destee A, et al. Tomographic measurements of regional cerebral blood flow in progressive supranuclear palsy and Parkinson's disease. Acta Neurol Scand 1995:92:235-41.

38 De Reuck J, Decoo D, Marchau M, et al. Positron emission tomography in vascular dementia [see comments]. J Neurol Sci 1998;154:55-61.

39 Sabri O, Hellwig D, Schreckenberger M, et al. Correlation of neuropsychological, morphological and functional (regional cerebral blood flow and glucose utilization) findings in cerebral microangiopathy. J Nucl Med 1998;39:147-54

40 Sultzer DL, Mahler ME, Cummings JL, et al. Cortical abnormalities associated with subcortical lesions in vascular dementia. Clinical and position emission tomographic findings. Arch Neurol 1995;52:773-80. 